\title{
Two-particle interferometry for the sources undergoing first-order QCD phase transition in high energy heavy ion collisions
}

\author{
Hong-Jie Yin, Jing Yang, and Wei-Ning Zhang* \\ School of Physics and Optoelectronic Technology, \\ Dalian University of Technology, Dalian, Liaoning 116024, China \\ $\mathrm{Li}-\mathrm{Li} \mathrm{Yu}$ \\ College of Science, Nanjing Forestry University, Nanjing, Jiangsu 210037, China
}

(Dated: November 28, 2018)

\begin{abstract}
We investigate the two-particle interferometry for the particle-emitting sources which undergo the first-order phase transition from the quark-gluon plasma with a finite baryon chemical potential to hadron resonance gas. The effects of source expansion, lifetime, and particle absorption on the transverse interferometry radii $R_{\text {out }}$ and $R_{\text {side }}$ are examined. We find that the emission durations of the particles become large when the system is initially located at the boundary between the mixed phase and the quark-gluon plasma. In this case, the difference between the radii $R_{\text {out }}$ and $R_{\text {side }}$ increases with the transverse momentum of the particle pair significantly. The ratio of $\sqrt{R_{\text {out }}^{2}-R_{\text {side }}^{2}}$ to the transverse velocity of the pair is an observable for the enhancement of the emission duration.

PACS numbers: 25.75.-q, 25.75.Gz, 25.75.Nq
\end{abstract}

\section{INTRODUCTION}

One of the important issues of high energy heavy ion collisions is to find and quantify the quantum chromodynamics $(\mathrm{QCD})$ phase transition between the quark-gluon plasma (QGP) at higher energy density and the hadron gas at lower energy density. The initial systems produced in the heavy ion collisions at the higher energies of the Super Proton Synchrotron (SPS) and Relativistic Heavy Ion Collider (RHIC) and the energy of the Large Hadron Collider (LHC) have high temperature and near-zero baryon chemical potential. Lattice QCD calculations have shown that the transition at the vanishing baryon chemical potential is a crossover [1]. However, it is predicted that this crossover will become a first-order phase transition at intermediate temperatures and high baryon chemical potentials [2 7]. Recently, the search for the evidences of the first-order phase transition and location of its critical end point have attracted special attention, for instance, the RHIC and SPS low energy programs [8 8 12] and the project of the future Facility for Antiproton and Ion Research (FAIR) at GSI [13-16].

Two-particle Hanbury-Brown-Twiss (HBT) interferometry is a useful tool for detecting the space-time structure of particle-emitting sources in high energy heavy ion collisions [17 20]. For the first-order phase transition there is a mixed phase of the QGP and hadron gas. In the absence of pressure gradient, a slow-burning fireball is expected when the initial system is at rest in the mixed phase, and this may lead to a considerable time-delay of the system evolution 21 26]. It is therefore of interest to probe the time-delay for the first-order phase transition by HBT interferometry.

\footnotetext{
*wnzhang@dlut.edu.cn
}

In Ref. 27] an HBT analysis technique with quantum transport of the interfering pair (QTIP) is developed. It takes into account the effects of resonance decay and multiple scattering of pions in the sources. In Ref. [28], this HBT technique is used to investigate the source radius and lifetime for the spherical systems evolving hydrodynamically with the first-order phase transition. In this study we use the relativistic hydrodynamics in $(2+1)$ dimension to describe the particle-emitting sources which undergo the first-order phase transition. We investigate the HBT radii $R_{\text {out }}, R_{\text {side }}$, and $R_{\text {long }}[29,30]$ for the hydrodynamic sources using the HBT interferometry with the QTIP technique. The results indicate that the ratio of $\Delta R_{\mathrm{os}}=\sqrt{R_{\text {out }}^{2}-R_{\text {side }}^{2}}$ to the transverse velocity of the particle pair $v_{\mathrm{KT}}$ is sensitive to the emission duration of the source. It is large when the system is initially located at the boundary of the mixed phase and the QGP (soft point). As compared to pion HBT interferometry kaon HBT interferometry may present more clearly the source space-time geometry at the emission, because kaons (for instance $\mathrm{K}^{+}$) can escape easily from the system after their production. By comparing the results of the two-pion and two-kaon HBT analyses, we find that the particle absorptions and the large expansion velocities of the sources after hadronization may change the pion HBT radii as functions of the transverse momentum of the particle pair. However, the large values of the ratio $\Delta R_{\mathrm{OS}} / v_{\mathrm{KT}}$ for the soft point of the first-order phase transition can be observed in both of two-pion and two-kaon HBT measurements.

The paper is organized as follows. In section II we present briefly the description for the relativistic hydrodynamics in cylindrical coordinate frame. We describe the model of the equation of state (EOS) of first-order phase transition used in our calculations. The adiabatic cooling paths and the space-time evolution of the systems are also discussed in this section. In section III we per- 
form the two-pion and two-kaon HBT analyses, with the QTIP technique, for the hydrodynamic particle-emitting sources for the initial conditions of the QGP and the soft point of the first-order phase transition. The effects of source expansion, lifetime, and particle absorptions on transverse HBT radii are investigated. On the basis of the investigations, we introduce an observable to probe the long lifetime of the source for the initial conditions of the soft point. Finally, the summary and conclusions are presented in section IV.

\section{HYDRODYNAMICAL EVOLUTION WITH FIRST-ORDER PHASE TRANSITION}

\section{A. Relativistic hydrodynamic equations in cylindrical frame}

The dynamics of ideal fluid in high energy heavy ion collisions is defined by the local conservations of energymomentum and net charges [31, 32]. The continuity equations of the conservations of energy-momentum, net baryon number, and entropy are

$$
\begin{gathered}
\partial_{\mu} T^{\mu \nu}(x)=0, \\
\partial_{\mu} j_{b}^{\mu}(x)=0, \\
\partial_{\mu} j_{s}^{\mu}(x)=0,
\end{gathered}
$$

where $x$ is the space-time coordinate of a thermalized fluid element in the source center-of-mass frame, $T^{\mu \nu}(x)$ is the energy momentum tensor of the element, $j_{b}^{\mu}(x)=$ $n_{b}(x) u^{\mu}$ and $j_{s}^{\mu}(x)=s(x) u^{\mu}$ are the four-current-density of baryon and entropy $\left(n_{b}\right.$ and $s$ are the baryon density and entropy density), and $u^{\mu}=\gamma(1, \boldsymbol{v})$ is the fourvelocity of the fluid element. The energy momentum tensor $T^{\mu \nu}(x)$ is given by [31, 32.

$$
T^{\mu \nu}(x)=[\varepsilon(x)+p(x)] u^{\mu}(x) u^{\nu}(x)-p(x) g^{\mu \nu},
$$

where $p$ and $\varepsilon$ are the pressure and energy density of the fluid element, and $g^{\mu \nu}$ is the metric tensor.

In the cylindrical coordinate $(t, \rho, \phi, z)$ frame, $g^{\mu \nu}=$ $\operatorname{diag}\left(1,-1,-\rho^{-2},-1\right)$. The conservation Eqs. (11) - (3) can be expressed as

$$
\begin{gathered}
\partial_{t} E+\partial_{\rho}\left[(E+p) v^{\rho}\right]+\partial_{z}\left[(E+p) v^{z}\right]=-\frac{v^{\rho}}{\rho}(E+p), \\
\partial_{t} M^{\rho}+\partial_{\rho}\left(M^{\rho} v^{\rho}+p\right)+\partial_{z}\left(M^{\rho} v^{z}\right)=-\frac{v^{\rho}}{\rho} M^{\rho}, \\
\partial_{t} M^{z}+\partial_{\rho}\left(M^{z} v^{\rho}\right)+\partial_{z}\left(M^{z} v^{z}+p\right)=-\frac{v^{\rho}}{\rho} M^{z}, \\
\partial_{t} N_{b}+\partial_{\rho}\left(N_{b} v^{\rho}\right)+\partial_{z}\left(N_{b} v^{z}\right)=-\frac{v^{\rho}}{\rho} N_{b},
\end{gathered}
$$

$$
\partial_{t} N_{s}+\partial_{\rho}\left(N_{s} v^{\rho}\right)+\partial_{z}\left(N_{s} v^{z}\right)=-\frac{v^{\rho}}{\rho} N_{s},
$$

where $E \equiv T^{00}, M^{\rho} \equiv T^{0 \rho}=T^{\rho 0}, M^{z} \equiv T^{0 z}=T^{z 0}$, $N_{b} \equiv j_{b}^{0}=n_{b} \gamma$, and $N_{s} \equiv j_{s}^{0}=s \gamma$.

\section{B. Equation of state}

In the equations of motion (5) - (9), there are $\varepsilon, p, v^{\rho}$, $v^{z}, n_{b}$, and $s$ six unknown functions. In order to obtain the solution of the equations of motion, we need an equation of state (EOS), $p\left(\varepsilon, n_{b}, s\right)$, which gives a relation for $p, \varepsilon, n_{b}$, and $s$. In our model the QGP phase is described by a perfect gas of gluons, $u, d, s$ quarks, and antiquarks, with the constant vacuum energy $B$ associated with QCD confinement 33]. The pressure, energy density, and the conserved charge density in the QGP phase are given by

$$
\begin{gathered}
p^{Q}=\sum_{i} p_{i}\left(T, \mu_{i}\right)-B, \\
\varepsilon^{Q}=\sum_{i} \varepsilon_{i}\left(T, \mu_{i}\right)+B, \\
n_{A}^{Q}=\sum_{i} A_{i} n_{i}\left(T, \mu_{i}\right),
\end{gathered}
$$

where $p_{i}\left(T, \mu_{i}\right), \varepsilon_{i}\left(T, \mu_{i}\right)$, and $n_{i}\left(T, \mu_{i}\right)$ are the pressure, energy density, and number density of particle species $i$ in the perfect gas with temperature $T$ and chemical potential $\left\{\mu_{i}\right\}, A_{i}$ is the conserved charge number of the particle species $i$. In our calculations we use the quark masses $m_{u}=m_{d}=5 \mathrm{MeV}, m_{s}=150 \mathrm{MeV}$ and the bag constant $B=(235 \mathrm{MeV})^{4} 33$.

For the hadronic phase we adopt the excluded volume model [33 35] and consider the particles $\pi, K, N, \Lambda, \Sigma$, $\Delta$, and their antiparticles in the model. The pressure, energy density, and the conserved charge density in the hadronic phase are given by [33 35$]$

$$
\begin{gathered}
p^{H}=\sum_{i} p_{i}\left(T, \tilde{\mu}_{i}\right), \\
\varepsilon^{H}=\frac{\sum_{i} \varepsilon_{i}\left(T, \tilde{\mu}_{i}\right)}{1+V_{0} \sum_{i} n_{i}\left(T, \tilde{\mu}_{i}\right)}, \\
n_{A}^{H}=\frac{\sum_{i} A_{i} n_{i}\left(T, \tilde{\mu}_{i}\right)}{1+V_{0} \sum_{i} n_{i}\left(T, \tilde{\mu}_{i}\right)},
\end{gathered}
$$

where

$$
\tilde{\mu}_{i}=\mu_{i}-V_{0} p^{H},
$$

$V_{0}=(1 / 2)(4 \pi / 3)(2 a)^{3}$ is the excluded volume which is assumed to be the same for all hadrons with $a=0.5 \mathrm{fm}$ [33]. 
For the first-order phase transition, there are Gibbs relationships in the mixed phase of the QGP and hadron gas. We have $T^{Q}=T^{H}, \mu_{N, \Delta}=3 \mu_{u}, \mu_{\Lambda, \Sigma}=2 \mu_{u}+\mu_{s}$, $\mu_{\pi^{+}, \pi^{0}, \pi^{-}}=0, \mu_{K^{+}, K^{0}}=\mu_{u}-\mu_{s}, \ldots$, and

$$
\begin{gathered}
p^{M}=p^{Q}\left(T, \mu_{u}, \mu_{s}\right)=p^{H}\left(T, \mu_{u}, \mu_{s}\right), \\
\varepsilon^{M}=\alpha \varepsilon^{Q}\left(T, \mu_{u}, \mu_{s}\right)+(1-\alpha) \varepsilon^{H}\left(T, \mu_{u}, \mu_{s}\right), \\
n_{A}^{M}=\alpha n_{A}^{Q}\left(T, \mu_{u}, \mu_{s}\right)+(1-\alpha) n_{A}^{H}\left(T, \mu_{u}, \mu_{s}\right),
\end{gathered}
$$

where $\mu_{u}$ and $\mu_{s}$ are the chemical potentials of $u$ and $s$ quarks, and $\alpha=V_{Q} / V$ is the fraction of the volume occupied by the plasma phase. The boundaries of the coexistence region are found by putting $\alpha=0$ (the hadron phase boundary) and $\alpha=1$ (the plasma boundary).

Using the thermodynamical relations of mixed gas one can get the entropy densities $s$ and other thermodynamical quantities, in the QGP, hadronic, and mixed phases from Eqs. [(10) - (12)], [(13) - (15)], and [(17)-(19)], and get numerically the EOS with the first-order phase transition.

\section{Adiabatic paths}

For perfect fluid, the entropy and baryon number of the system are conserved during evolution. So the ratio of the densities $n_{b}$ and $s, n_{b} / s$, is a constant. In the calculations we take $n_{b} / s=0.06$ which corresponds to the incident energy about $30 \mathrm{AGeV}[36]$. The solid lines in Fig. 1 show the adiabatic cooling paths for the system evolving with the EOS of the first-order phase transition. The dotted line is the transition curve between the QGP and hadron gas. The mixed phase is on the transition curve from the end point of the QGP branch (point 1) to the beginning of the hadronic branch (point 2). The non-trivial zigzag shape of the trajectory indicates that the system has a re-heating in the mixed phase [35, 37]. The reason is that at a certain point $(T, \mu)$ on the phasetransition curve, the number of degrees of freedom, and hence the specific entropy, is larger in the QGP phase than which in the hadronic phase. The temperature must increase during hadronization to conserve both the total entropy and baryon number simultaneously [37].

In Fig. 2 we show the thermodynamical quantity, $p / \varepsilon$, as a function of $\varepsilon$ for the system. The ratio $p / \varepsilon$ reaches the minimum at the boundary between the QGP and mixed phase, $\varepsilon=\varepsilon^{\mathrm{MQ}}=1.83 \mathrm{GeV} / \mathrm{fm}^{3}$. It is so called the soft point of the first-order phase transition. At the boundary between the mixed phase and hadronic gas, the ratio reaches its maximum. It is named hadronization point. One can see that the ratio retains the values smaller than 0.075 in the $\varepsilon$ regain $0.6-2.1 \mathrm{GeV} / \mathrm{fm}^{3}$.

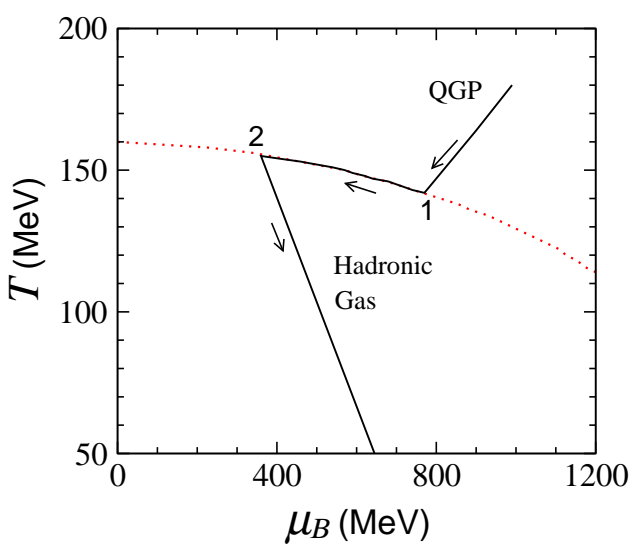

FIG. 1: (Color online) The adiabatic paths for the system evolving with the EOS of the first-order phase transition. The dotted line is the transition curve between the QGP and hadron gas. The mixed phase is on the transition curve from the end point of the QGP branch (point 1) to the beginning of the hadronic branch (point 2).

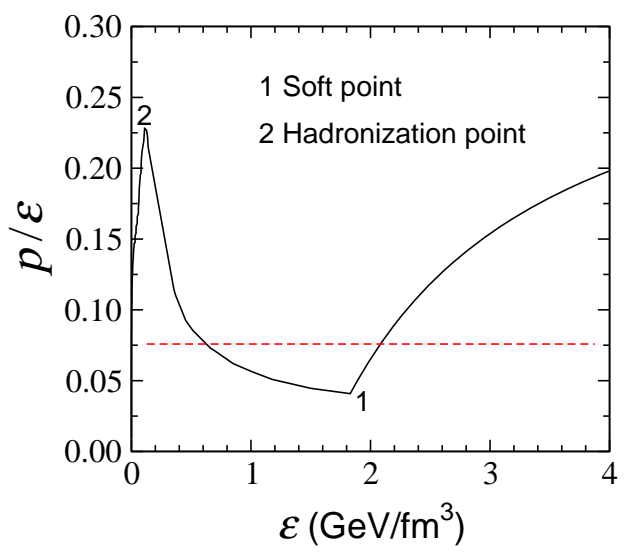

FIG. 2: (Color online) The ratio of the system pressure to energy density, $p / \varepsilon$. It has the minimum at the soft point 1 and reaches its maximum at the hadronization point 2 . The ratio retains the values smaller than 0.075 in the $\varepsilon$ regain 0.6 $-2.1 \mathrm{GeV} / \mathrm{fm}^{3}$

\section{System evolution}

Using the Sod's operator splitting and RHLLE method 31, 38-40], we can obtain the system evolution by solving the hydrodynamical equations (5) - (9) with the EOS of the first-order phase transition. Because the heavy ion collisions are full stopped at the energy considered, we assume the system is initially at rest within a cylinder in the beam direction ( $z$-direction) with the transverse and longitudinal radii $\rho_{0}$ and $z_{0}$. In Fig. 3, we show the two-dimension energy density, $\varepsilon(x, z)=\int \varepsilon(x, y, z) d y$, for the systems at the time $t=0,6$, and $12 \mathrm{fm} / \mathrm{c}$. The left and right panels are for the systems which are initially located in the QGP phase $\left(T_{0}^{\mathrm{QGP}}=180 \mathrm{MeV}, \varepsilon_{0}^{\mathrm{QGP}}=\right.$ $\left.4.12 \mathrm{GeV} / \mathrm{fm}^{3}, \mu_{B 0}^{\mathrm{QGP}}=990 \mathrm{MeV}\right)$ and at the soft point 

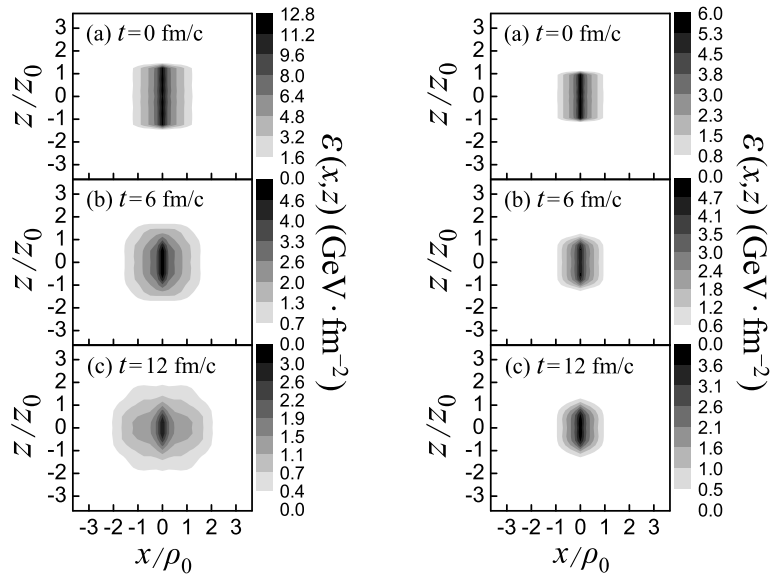

FIG. 3: The two-dimension energy density, $\varepsilon(x, z)$, for the systems at the time $t=0,6$, and $12 \mathrm{fm} / \mathrm{c}$. The left panels are for the system initially in the QGP. The right panels are for the system initially at the soft point.

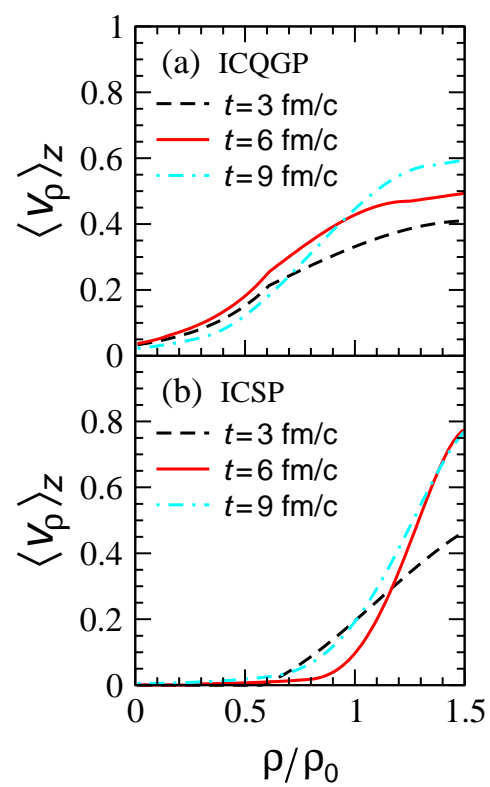

FIG. 4: (Color online) The average transverse velocity for the systems initially in the QGP (a) and at the soft point (b).

$\left(T_{0}^{\mathrm{SP}}=142 \mathrm{MeV}, \varepsilon_{0}^{\mathrm{SP}}=\varepsilon^{\mathrm{MQ}}=1.83 \mathrm{GeV} / \mathrm{fm}^{3}, \mu_{B 0}^{\mathrm{SP}}=\right.$ $780 \mathrm{MeV}$ ). It can be seen that the energy density for the system with the initial conditions of the soft point (ICSP) decreases more slowly with time than that for the system with the initial conditions of the QGP (ICQGP). Because there are not the initial velocity and pressure gradient in the mixed phase, the expansion of the system is slow. Figure 4 (a) and (b) show the average transverse velocity, $\left\langle v_{\rho}(\rho)\right\rangle_{z}=\int v_{\rho}(\rho, z) d z$, for the systems with ICQGP and ICSP, respectively. For ICQGP, the velocity increases rapidly from zero at the beginning $(t \leq 3 \mathrm{fm} / \mathrm{c})$, and still increases with time during $3<t<6 \mathrm{fm} / \mathrm{c}$.
Because there is larger gradient of pressure on the edge of the system, the velocity increase more rapidly around $\rho \sim \rho_{0}$. At $t=9 \mathrm{fm} / \mathrm{c}$, the decrease of the velocity near the center of the system is due to the blast-wave expansion which leads to a void in the center region. For ICSP, the velocity retains zero in the center region of the system even at a larger time because there is not pressure gradient in this case. In our calculations, the initial sizes for the system with ICQGP are taken to be $\rho_{0}=z_{0}=4.0$ $\mathrm{fm}$. The initial sizes for the system with ICSP are taken to be $\rho_{0}=z_{0}=4.0 \times\left(\varepsilon_{0}^{\mathrm{QGP}} / \varepsilon^{\mathrm{MQ}}\right)^{1 / 3}=5.2 \mathrm{fm}$.

\section{HBT INTERFEROMETRY WITH QUANTUM TRANSPORT OF THE INTERFERING PAIR}

\section{A. Formulas of correlation function}

The two-particle HBT correlation function $C\left(k_{1}, k_{2}\right)$ is defined as the ratio of the two-particle momentum distribution $P\left(k_{1}, k_{2}\right)$ to the product of the single-particle momentum distribution $P\left(k_{1}\right) P\left(k_{2}\right)$,

$$
C\left(k_{1}, k_{2}\right)=\frac{P\left(k_{1}, k_{2}\right)}{P\left(k_{1}\right) P\left(k_{2}\right)} .
$$

Using the quantum probability amplitudes in a pathintegral formalism [27], $P\left(k_{i}\right)\left(k_{i}=\left(E_{i}, \mathbf{k}_{i}\right), i=1,2\right)$ and $P\left(k_{1}, k_{2}\right)$ can be expressed as [27, 41,44$]$

$$
\begin{aligned}
P\left(k_{i}\right)= & \int d^{4} x \rho(x) e^{-2 \mathcal{I} m \bar{\phi}_{s}\left(x \kappa \rightarrow k_{i}\right)}|A(x \kappa)|^{2} \\
P\left(k_{1}, k_{2}\right)= & \int d^{4} x_{1} d^{4} x_{2} e^{-2 \mathcal{I} m \bar{\phi}_{s}\left(x_{1} \kappa_{1} \rightarrow k_{1}\right)} e^{-2 \mathcal{I} m \bar{\phi}_{s}\left(x_{2} \kappa_{2} \rightarrow k_{2}\right)} \\
& \times \rho\left(x_{1}\right) \rho\left(x_{2}\right)\left|\Phi\left(x_{1} x_{2} ; k_{1} k_{2}\right)\right|^{2}
\end{aligned}
$$

where $\rho(x)$ is the four-dimension density of the particleemitting source, $A(x \kappa)$ is the amplitude for producing a particle at $x$ with momentum $\kappa, e^{-2 \operatorname{Im} \bar{\phi}_{s}(x \kappa \rightarrow k)}$ is the absorption factor due to the multiple scattering when the particle propagating in the source, and $\Phi\left(x_{1} x_{2} ; k_{1} k_{2}\right)$ is the wave function for the two identical bosons,

$$
\begin{aligned}
& \Phi\left(x_{1} x_{2} ; k_{1} k_{2}\right)= \\
& \quad \frac{1}{\sqrt{2}}\left\{\bar{A}\left(x_{1} \kappa_{1}, k_{1}\right) \bar{A}\left(x_{2} \kappa 2, k_{2}\right) e^{i k_{1} \cdot x_{1}+i k_{2} \cdot k_{2}}\right. \\
& \quad+\left\{\bar{A}\left(x_{1} \kappa_{2}^{\prime}, k_{2}\right) \bar{A}\left(x_{2} \kappa_{1}^{\prime}, k_{1}\right) e^{i k_{1} \cdot x_{1}+i k_{2} \cdot k_{2}}\right\} \\
& \bar{A}(x \kappa, k)=A(x \kappa) e^{i \delta_{\mathrm{mf}}(x \kappa \rightarrow k)}
\end{aligned}
$$

where $\delta_{\mathrm{mf}}(x \kappa \rightarrow k)$ is a phase arising from the source collective expansion, which can be described by a longrange density-dependent mean-field [27, 44].

In our HBT calculations, the identical kaons (for instance $\mathrm{K}^{+}$) are assumed to freeze out directly at the 
hadronization. So, the absorption factor $e^{-2 \operatorname{Im} m \bar{\phi}_{s}}$ is 1 and $\delta_{\mathrm{mf}}=0$. The final identical pions (for instance $\pi^{+}$) include the primary pions emitted at the hadronization and the secondary pions from the "excited-state" particle decays during the system evolving in hadronic phase until to the thermal freeze-out. The four-dimension density of the pion source can be expressed as $27,45,46]$

$$
\rho(x)=n_{\pi}(x) \delta\left(t-\tau^{h}\right)+\sum_{j \neq \pi} D_{j \rightarrow \pi} n_{j}(x),
$$

where $n_{i}(x)$ and $\tau^{h}$ are the particle number density and the hadronization time in local frame, $D_{j \rightarrow \pi}$ is the product of the decay rate in time and the fraction of the decay. For example, $D_{\Delta \rightarrow \pi}=\Gamma_{\Delta} \times \frac{1}{3}$ and $D_{\pi^{0} \pi^{0} \rightarrow \pi^{+} \pi^{-}}=$ $v_{r} n_{\pi} \sigma\left(\pi^{0} \pi^{0} \rightarrow \pi^{+} \pi^{-}\right) \times 1$, where $v_{r}$ is the relative velocity of the two colliding pions and the cross section $\sigma\left(\pi^{0} \pi^{0} \rightarrow \pi^{+} \pi^{-}\right)$is equal to the absorption cross section of $\pi^{+} \pi^{-} \rightarrow \pi^{0} \pi^{0}$ [27].

When a pion propagating in the source it will subject to multiple scattering with the medium particles in the source. The absorption factor due to the multiple scattering in Eqs. (21) and (22) can be written as [27, 41 44]

$$
e^{-2 \mathcal{I} m \bar{\phi}_{s}(x)}=\exp \left[-\int_{x}^{x_{f}}\left(\sum_{i}^{\prime} \sigma_{\mathrm{abs}}(\pi i) n_{i}\left(x^{\prime}\right)\right) d \ell\left(x^{\prime}\right)\right],
$$

where $\sum_{i}^{\prime}$ means the summation for all medium particles except for the test pion along the propagating path $d \ell\left(x^{\prime}\right), \sigma_{\text {abs }}(\pi i)$ is the absorption cross section of the pion with the particle species $i$ in the medium, and $x_{f}$ is the freeze-out coordinate. In calculations we only consider the dominant absorption processes for the identical pions, for example the reactions of $\pi^{+} \pi^{-} \rightarrow \pi^{0} \pi^{0}$ and $\pi^{+} N \rightarrow \Delta$ for $\pi^{+}$, as in Ref. [27]. The pion freeze-out temperature is taken to be $110 \mathrm{MeV}$, which corresponds to the energy density $\varepsilon_{f}=45 \mathrm{MeV} / \mathrm{c}$ [47].

In the HBT analysis, we use the Bertsch-Pratt components of the relative momentum $q=\left|\mathbf{k}_{1}-\mathbf{k}_{2}\right|$ of the identical particle pair [29, 30], $q_{\text {side }}, q_{\text {out }}$, and $q_{\text {long }}$ as variables. The correlation function $C_{K}\left(q_{\text {side }}, q_{\text {out }}, q_{\text {long }}\right)$ are constructed from $P\left(k_{1}, k_{2}\right)$ and $P\left(k_{1}\right) P\left(k_{2}\right)$ by summing over $\mathbf{k}_{1}$ and $\mathbf{k}_{2}$ for the $\left(q_{\text {side }}, q_{\text {out }}, q_{\text {long }}\right)$ bins in a certain $K_{T}=\frac{1}{2}\left|\mathbf{k}_{1}-\mathbf{k}_{2}\right|_{T}$ region. The HBT radii $R_{\text {side }}\left(K_{T}\right)$, $R_{\text {out }}\left(K_{T}\right)$, and $R_{\text {long }}\left(K_{T}\right)$ are obtained by fitting the correlation functions with the parametrized formula

$$
\begin{gathered}
C_{K}\left(q_{\text {side }}, q_{\text {out }}, q_{\text {long }}\right)=1+\lambda \exp \left[-q_{\text {side }}^{2} R_{\text {side }}^{2}\left(K_{T}\right)\right. \\
\left.-q_{\text {out }}^{2} R_{\text {out }}^{2}\left(K_{T}\right)-q_{\text {long }}^{2} R_{\text {long }}^{2}\left(K_{T}\right)\right]
\end{gathered}
$$

in the longitudinal comoving system (LCMS). Here $\lambda$ is called the chaotic parameter.

\section{B. Results for hydrodynamic sources}

In Fig. 5 we show the two-pion and two-kaon HBT results for the hydrodynamic sources for ICQGP and ICSP.

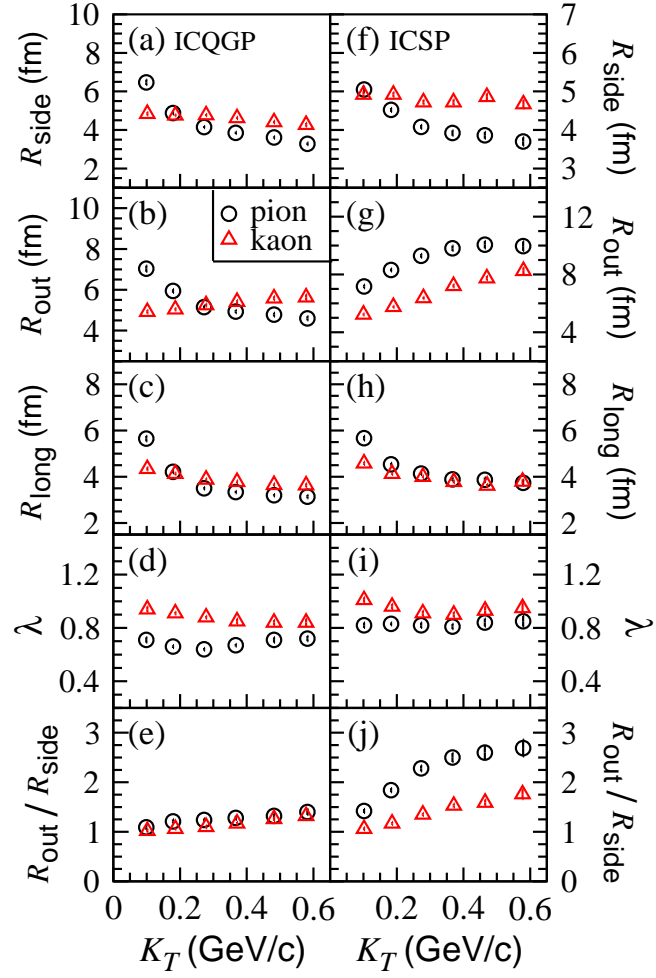

FIG. 5: (Color online) The two-pion and two-kaon HBT results for the hydrodynamic sources for ICQGP and ICSP.

It can be seen that there is much difference for the twopion HBT radius $R_{\text {out }}$ as functions of $K_{T}$ for the two kinds of sources. One decreases with $K_{T}$, and another almost increase with $K_{T}$. When the system is initially located at the soft point (ICSP case), the results of $R_{\text {out }}$ are much larger than those of $R_{\text {side }}$ at larger $K_{T}$, and the ratio $R_{\text {out }} / R_{\text {side }}$ increases with $K_{T}$ significantly. As compared to the pion HBT radii the kaon HBT radii exhibit more moderate changes with $K_{T}$.

Figure 6 (a) and (b) show the transverse velocities of the pion- and kaon-emitting sources as functions of the pair transverse momenta $K_{T}$. Figure 6 (c) and (d) show the standard deviations of time, $\sigma_{t}=\sqrt{\left\langle(t-\langle t\rangle)^{2}\right\rangle}$, of the particle-emitting sources. One can see that the transverse velocities of the pion and kaon sources are smaller for the system initially at the soft point (ICSP) than those for the system initially in the QGP (ICQGP). The standard deviations of time enhance very much for the sources for ICSP.

In HBT interferometry, the source HBT radii are related to the enhancements of the correlation functions at small relative momenta. For an evolving source, the source expansion leads to a correlation between the particle-emitting coordinate and momentum. It may decrease the transverse emission region for the particle pairs with small relative momenta and large $K_{T}$. This effect is more important in the direction of the transverse momentum of the pair (out direction), which is boosted by the source expansion. Additionally, the source opacity, due 


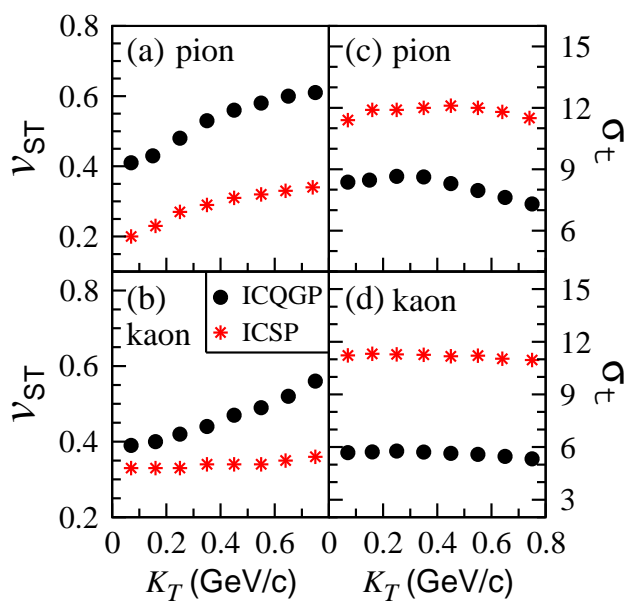

FIG. 6: (Color online) Left panels: the transverse velocities of the particle-emitting sources as functions of $K_{T}$. Right panels: the standard deviations of time of the particles-emitting sources.

to the absorptions for the particles propagating through the center of source (in which the temperatures are higher than the hadronization temperature) and by the multiple scattering among the particles in the source, may lead to a shell emission. This will increase the effect of the decrease of emission region for expanding sources. In Fig 7. we show the distributions of the source coordinates projected on the transverse out-side plane, for the particles with the smaller pair momenta $K_{T}<300 \mathrm{MeV} / \mathrm{c}$ (left panels) and the larger pair momentum $K_{T}>300 \mathrm{MeV} / \mathrm{c}$ (right panels). The upper four panels are for the system initially in the QGP (ICQGP). The lower four panels are for the system initially at the soft point (ICSP). For $K_{T}>300 \mathrm{MeV} / \mathrm{c}$, the distributions of the source coordinates are more concentrated in $r_{o}>0$ regions. For $K_{T}<300 \mathrm{MeV} / \mathrm{c}$, the annular distributions for kaon indicate that the sources are almost transparent for the kaons emitted later. We will see it is that the source expansion, lifetime $\left(\sim \sigma_{t}\right)$, and particle absorptions lead to the differences of the transverse HBT radii $R_{\text {out }}$ for the two kinds of sources for ICQGP and ICSP.

\section{The effects of source expansion and lifetime on transverse HBT radii}

In HBT interferometry, the difference of the transverse HBT radii in out and side directions includes the important information on the source expansion and lifetime. $R_{\text {out }}^{2}-R_{\text {side }}^{2}$ is given by $[18,48,49$ ]

$$
R_{\text {out }}^{2}-R_{\text {side }}^{2}=\left\langle\left(\tilde{r}_{o}-v_{\mathrm{KT}} \tilde{t}\right)^{2}\right\rangle-\left\langle\tilde{r}_{s}^{2}\right\rangle,
$$

where $\langle\cdots\rangle$ denotes the average for the space-time coordinates of the source, $\tilde{r}_{o}$ and $\tilde{r}_{s}$ are the biases of the source spatial coordinates related to their average values in the out and side directions, $\tilde{t}$ is the bias of the source

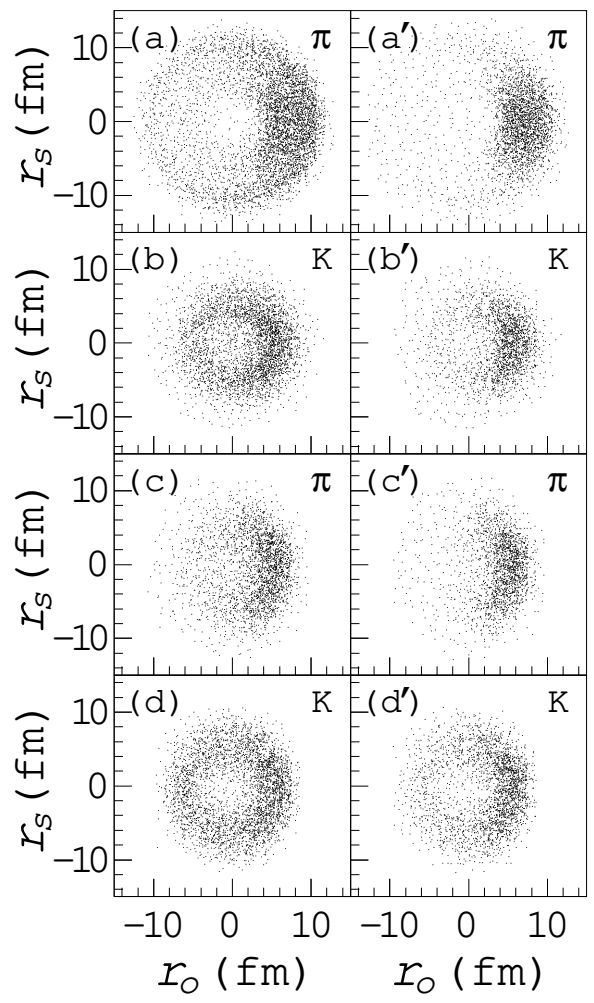

FIG. 7: The distributions of the source coordinates projected on the transverse out-side plane. The left and right panels are for the smaller and larger particle pair momenta $K_{T}<300$ $\mathrm{MeV} / \mathrm{c}$ and $K_{T}>300 \mathrm{MeV} / \mathrm{c}$. The upper four panels are for ICQGP. The lower four panels are for ICSP.

time coordinate related to its average, and $v_{\mathrm{KT}}$ is the transverse velocity of the particle pair.

In order to examine the effects of source expansion and lifetime on the transverse HBT radii $R_{\text {out }}$ and $R_{\text {side, }}$, we investigate next the two-pion interferometry for the simple sources with a constant temperature $100 \mathrm{MeV}$ and the Gaussian space-time distributions as

$$
\begin{gathered}
\frac{d N}{d^{3} r d t} \propto \exp \left(-\frac{x^{2}+y^{2}}{2 R_{T}^{2}}-\frac{z^{2}}{2 R_{L}^{2}}-\frac{t^{2}}{2 \tau^{2}}\right), \\
R_{1} \leq \sqrt{x^{2}+y^{2}+z^{2}} \leq R_{2} .
\end{gathered}
$$

We take $R_{T}=R_{L}=5 \mathrm{fm}, R_{2}=10 \mathrm{fm}$, and assume that the sources have the radial velocity

$$
v_{r}=v_{0} \frac{r}{R_{2}} .
$$

Here $\tau, R_{1}$, and $v_{0}$ are three free parameters. We taken $\tau=6$ and $12 \mathrm{fm} / \mathrm{c}$ for the sources with shorter and longer lifetimes. For a shell source $R_{1}$ is taken to be $5 \mathrm{fm}$. For static and expanding sources, $v_{0}$ is taken to be 0 and 0.8 respectively. Because there are not correlations between spatial coordinates and time for these sources, Eq. (28) reduces to

$$
R_{\mathrm{out}}^{2}-R_{\text {side }}^{2}=\left\langle\tilde{r}_{o}^{2}\right\rangle-\left\langle\tilde{r}_{s}^{2}\right\rangle+\sigma_{t}^{2} v_{\mathrm{KT}}^{2},
$$


where $\sigma_{t}^{2}=\left\langle(t-\langle t\rangle)^{2}\right\rangle=(\pi-2) \tau^{2} / \pi \approx 0.363 \tau^{2}$.

Because of source expansion and opacity the difference of the variances in out and side directions, $\left\langle\tilde{r}_{o}^{2}\right\rangle-\left\langle\tilde{r}_{s}^{2}\right\rangle$, is not zero even for the source with transverse symmetry. It is negative and decrease with $K_{T}$. On the other hand, the right third term in Eq. (31), $\sigma_{t}^{2} v_{\mathrm{KT}}^{2}$, is positive. It increases with $K_{T}$ and becomes important when the source lifetime $\tau$ increases.

In Fig. 8, we show the transverse HBT radii $R_{\text {out }}$ and $R_{\text {side }}$ and $\Delta R_{\text {os }}=\sqrt{R_{\text {out }}^{2}-R_{\text {side }}^{2}}$ for the sources with $\tau=6$ and $12 \mathrm{fm}$. The symbols $\circ, \nabla$, and $\Delta$ are for the static Gaussian source $\left(v_{0}=0, R_{1}=0 \mathrm{fm}\right)$, expanding Gaussian source $\left(v_{0}=0.8, R_{1}=0 \mathrm{fm}\right)$, and expanding shell source $\left(v_{0}=0.8, R_{1}=5 \mathrm{fm}\right)$. The dashed lines in the bottom panels are the results of $\sigma_{t} v_{\mathrm{KT}}$ $\left(v_{\mathrm{KT}}=K_{T} / E_{K}, E_{K}=\left(E_{1}+E_{2}\right) / 2\right.$. $)$ In Fig. 9, we show the distributions of the source coordinates projected on $r_{o}-r_{s}$ plane. The panels (a), (b), and (c) are for the static Gaussian source, expanding Gaussian source, and expanding shell source for the smaller pion pair momentum $K_{T}<300 \mathrm{MeV} / \mathrm{c}$. The panels $\left(\mathrm{a}^{\prime}\right),\left(\mathrm{b}^{\prime}\right)$, and $\left(\mathrm{c}^{\prime}\right)$ are for the static Gaussian source, expanding Gaussian source, and expanding shell source for $K_{T}>300 \mathrm{MeV} / \mathrm{c}$. For the static sources, the results of $R_{\text {side }}$ are almost a constant and $R_{\text {out }}$ increases with $K_{T}$. Because there is not the effect of source expansion, $\left\langle\tilde{r}_{o}^{2}\right\rangle=\left\langle\tilde{r}_{s}^{2}\right\rangle$, and the results of $\Delta R_{\mathrm{os}}$ are consistent with those of $\sigma_{t} v_{\mathrm{KT}}$. For the expanding and shell-emitting sources, the source expansion and shell emission change the distributions of the source coordinates. It leads to the decreases of $R_{\text {side }}$ with $K_{T}$. Although $R_{\text {out }}$ increases with $K_{T}$ in the small $K_{T}$ region for the sources with larger lifetime $\tau$, this increase will be counteracted at large $K_{T}$ by the effects of the source expansion and shell emission. In these cases, the results of $\Delta R_{\mathrm{os}}$ are smaller than the values of $\sigma_{t} v_{\mathrm{KT}}$ at larger $K_{T}$. From Fig. 9 one can see directly that the coordinate distributions of the static sources for the smaller and larger pion pair momenta are almost the same. However, the coordinate distributions of the expanding sources for $K_{T}>300 \mathrm{MeV} / \mathrm{c}$ are more concentrated in $r_{o}>0$ regions as compared to the corresponding distributions for $K_{T}<300 \mathrm{MeV} / \mathrm{c}$.

For hydrodynamic sources, there are also correlations between source spatial coordinates and time. We will discuss the effect of the correlation between $r_{o}$ and $t$ on $\Delta R_{\mathrm{os}}$ in next subsection.

\section{Characteristic quantity for long source lifetime for ICSP}

Because of the correlation between source spatial coordinate $r_{o}$ and time for hydrodynamic sources, Eq. (28) becomes

$$
R_{\text {out }}^{2}-R_{\text {side }}^{2}=\left\langle\tilde{r}_{o}^{2}\right\rangle-\left\langle\tilde{r}_{s}^{2}\right\rangle+\sigma_{t}^{2} v_{\mathrm{KT}}^{2}-2\left\langle\tilde{r}_{o} \tilde{t}\right\rangle v_{\mathrm{KT}} .
$$

For positive or negative $\left\langle\tilde{r}_{o} \tilde{t}\right\rangle$, the right last term in Eq. (32) will decrease or increase $\Delta R_{\mathrm{os}}$ with $K_{T}$ increase.

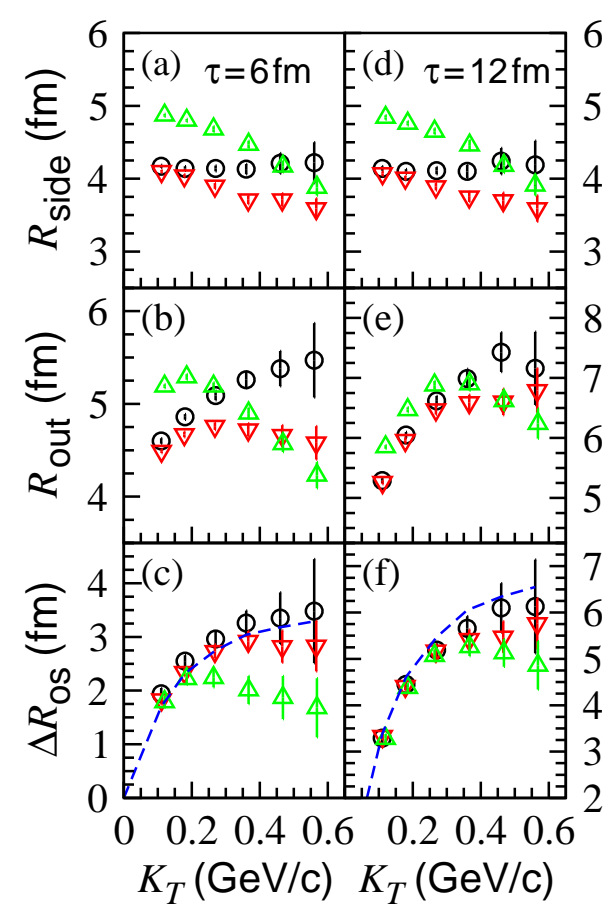

FIG. 8: (Color online) The transverse HBT radii and $\Delta R_{\mathrm{os}}=$ $\sqrt{R_{\text {out }}^{2}-R_{\text {side }}^{2}}$ for the static Gaussian source (circle), expanding Gaussian source (triangle down), and expanding shell source (triangle up). The dashed lines are the results of $\sigma_{t} v_{\mathrm{KT}}$.

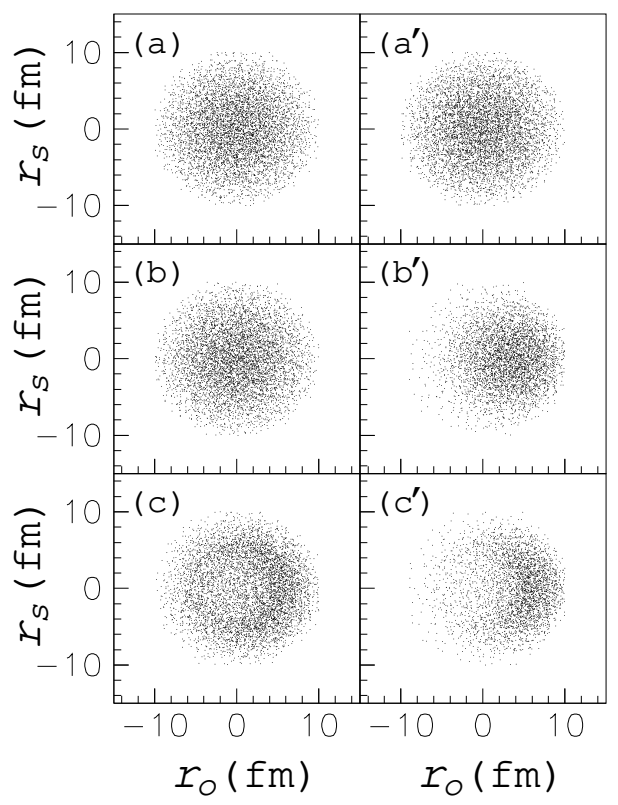

FIG. 9: The distributions of the source coordinates projected on $r_{o}-r_{s}$ plane. The panels (a), (b), and (c) are for the static Gaussian, expanding Gaussian, and expanding shell sources for $K_{T}<300 \mathrm{MeV} / \mathrm{c}$. The panels $\left(\mathrm{a}^{\prime}\right),\left(\mathrm{b}^{\prime}\right)$, and $\left(\mathrm{c}^{\prime}\right)$ are for the static Gaussian, expanding Gaussian, and expanding shell sources for $K_{T}>300 \mathrm{MeV} / \mathrm{c}$. 


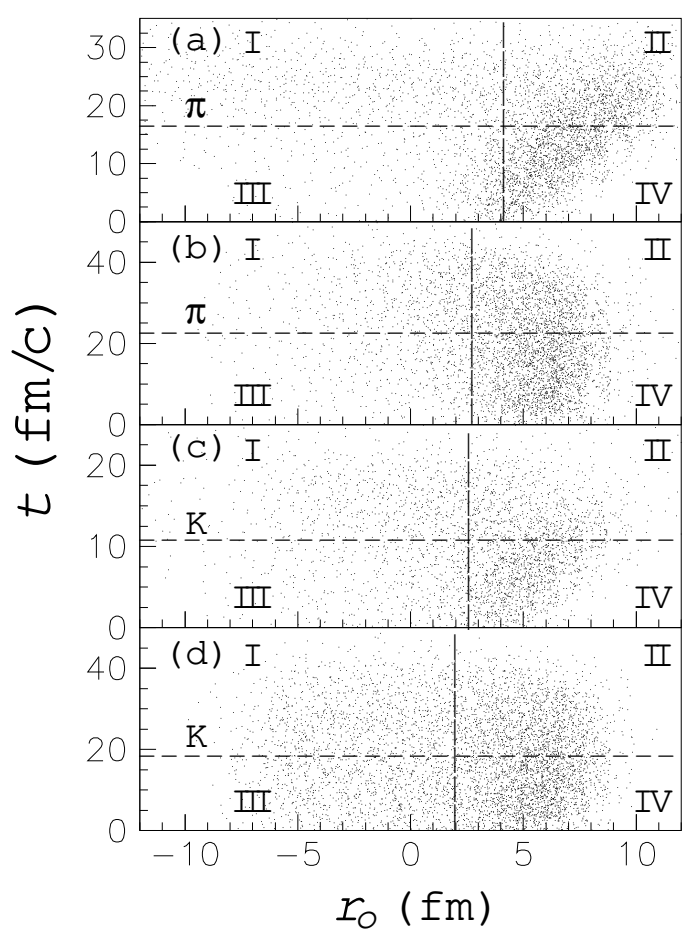

FIG. 10: The distributions of the space-time coordinates of source points projected on $r_{o}-t$ plane for ICQGP ((a) and (c)) and ICSP ((b) and (d)). The dashed lines are for the average values of $r_{o}$ and $t$.

In Fig. 10 we show the distributions of the space-time coordinates of source points projected on $r_{o}-t$ plane for ICQGP (panels (a) and (c)) and ICSP (panels (b) and (d)). The dashed lines are for the average values of $r_{o}$ and $t$, which divide the plane into four regions. In regions I and IV, $\left\langle\tilde{r}_{o} \tilde{t}\right\rangle<0$. In regions II and III, $\left\langle\tilde{r}_{o} \tilde{t}\right\rangle>0$. For pion, because of particle absorption the distributions in region III are less than those in region I. So the values of $\tilde{r}_{o} \tilde{t}$ averaging over all $r_{o}<\left\langle r_{o}\right\rangle$ regions (I and III) are negative. In $\left.r_{o}\right\rangle\left\langle r_{o}\right\rangle$ regions, the distribution for the pion source for ICQGP (panel (a)) is much different from that for ICSP (panel (b)) because of the larger transverse expansion of source for ICQGP. In region II of the panel (a), the wider distribution for the pion source for ICQGP leads to a greater contribution to $\left\langle\tilde{r}_{o} \tilde{t}\right\rangle$. So the value of $\tilde{r}_{o} \tilde{t}$ averaging over all $\left.r_{o}\right\rangle\left\langle r_{o}\right\rangle$ regions (II and IV) is positive for ICQGP. For ICSP, the distribution for pion source in region II is less than that in region IV. The value of $\tilde{r}_{o} \tilde{t}$ averaging over all $\left.r_{o}\right\rangle\left\langle r_{o}\right\rangle$ regions is also negative for ICSP. The calculations indicate that for pion $\left\langle\tilde{r}_{o} \tilde{t}\right\rangle=0.16$ and $-8.30 \mathrm{fm}^{2} / \mathrm{c}$ for ICQGP and ICSP, which are consistent with the above discussions. For kaon, because of the high transparency and low transverse expansion of sources, the distributions are approximately symmetric about $\langle t\rangle$. The values of $\left\langle\tilde{r}_{o} \tilde{t}\right\rangle$ are small for the kaon sources.

In Fig. 11 we show the results of $\Delta R_{\mathrm{os}}$ (symbols $\circ$ ) and the products $\bar{\sigma}_{t} v_{\mathrm{KT}}$ (symbols $*$ ) of the average emis-

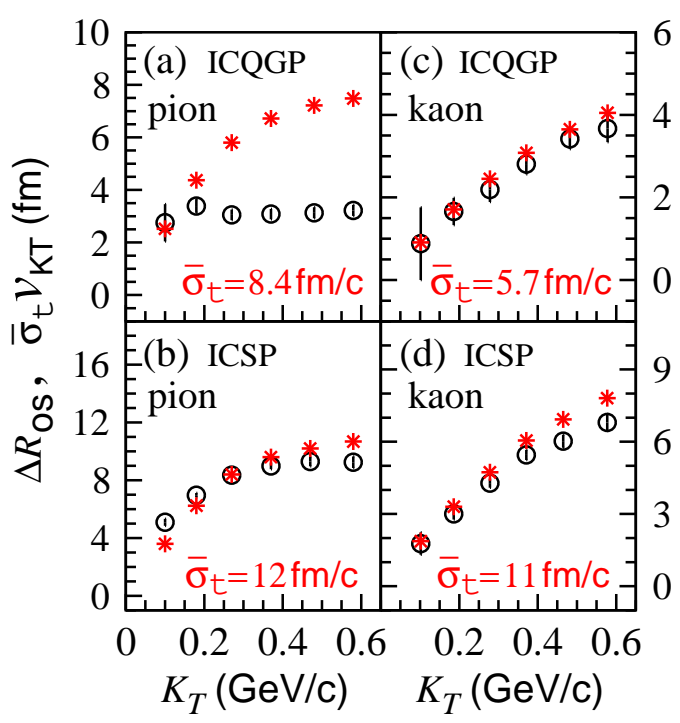

FIG. 11: (Color online) The results of $\Delta R_{\mathrm{Os}}(\mathrm{o})$ and $\bar{\sigma}_{t} v_{\mathrm{KT}}$ (*) for the hydrodynamic sources for ICQGP and ICSP.

sion durations $\bar{\sigma}_{t}$ of the particles and the pair transverse velocities $v_{\mathrm{KT}}$ for the hydrodynamic sources for ICQGP and ICSP. It can be seen that except for the pion results in panel (a), the results of the $\Delta R_{\mathrm{OS}}$ and $\bar{\sigma}_{t} v_{\mathrm{KT}}$ are almost consistent.

Inspired by the consistences of the results of $\Delta R_{\mathrm{OS}}$ and $\bar{\sigma}_{t} v_{\mathrm{KT}}$ for ICSP, we introduce the quantity

$$
\tilde{\sigma}=\frac{\Delta R_{\mathrm{os}}}{v_{\mathrm{KT}}}=\frac{\sqrt{R_{\mathrm{out}}^{2}-R_{\mathrm{side}}^{2}}}{K_{T} / E_{K}}
$$

to describe the character of the long lifetime of the sources for ICSP. It is an experimental observable.

In Fig. 12 we show the results of $\tilde{\sigma}$ for pion and kaon for the hydrodynamic sources for ICQGP and ICSP. The larger values of $\tilde{\sigma}$ for the soft point of the first-order phase transition are observed in both of the pion and kaon interferometry measurements. The $\tilde{\sigma}$ values for the pion source for ICQGP are much smaller than the average value $\bar{\sigma}_{t}=8.4 \mathrm{fm} / \mathrm{c}$ at large $K_{T}$, because of the large transverse velocities of the source and the positive values of $\left\langle\tilde{r}_{o} \tilde{t}\right\rangle$. In this case $\tilde{\sigma}$ cannot reflect the real lifetime of the source. At small $K_{T}$, the larger values of $\tilde{\sigma}$ for the pion source for ICSP are due to the large negative values of $\left\langle\tilde{r}_{o} \tilde{t}\right\rangle$ as well as the small transverse velocities of the source in this case. The errors of $\tilde{\sigma}$ exhibited in Fig. 12 are only from the statistic errors of $R_{\text {out }}$ and $R_{\text {side }}$ related to the HBT parametrized fits. In high energy heavy ion collisions, there are other effects which may bring uncertainty to the observable, for example the non-equilibrium dynamics during the decay of resonances after the hadronization. Further investigations on these effects will be of interest. 


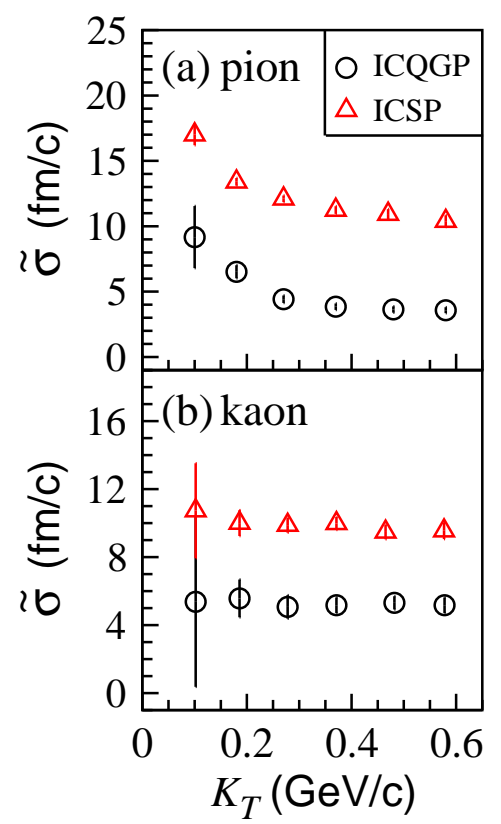

FIG. 12: (Color online) The results of $\tilde{\sigma}$ for the hydrodynamic sources for ICQGP and ICSP.

\section{SUMMARY AND CONCLUSIONS}

We investigate the two-particle HBT interferometry for the hydrodynamic particle-emitting sources which undergo the first-order phase transition from the quarkgluon plasma with finite baryon chemical potentials to hadron resonance gas. The effects of source expansion, lifetime, and particle absorption on the HBT radii are examined. For pion, the large transverse expansion of the source for ICQGP decreases the HBT radii $R_{\text {out }}$ and $R_{\text {side }}$ at large transverse momentum of particle pair $K_{T}$. The source has a long lifetime and small expansion when the system is initially located at the boundary between the mixed phase and the QGP (soft point). In this case, the difference between the transverse HBT radii $R_{\text {out }}$ and $R_{\text {side }}$ increases with $K_{T}$ significantly. The ratio of $\sqrt{R_{\text {out }}^{2}-R_{\text {side }}^{2}}$ to the transverse velocity of the particle pair $v_{\mathrm{KT}}, \tilde{\sigma}$, is an observable for probing the long lifetime of the source for the soft point of the first-order phase transition. As compared to pion HBT interferometry kaon HBT interferometry may present more clearly the source space-time geometry at the emission. The larger values of $\tilde{\sigma}$ for the soft point of the first-order phase transition can be observed both by two-pion and two-kaon HBT measurements. Further investigations on other effects on the observable will be of interest.

\section{Acknowledgments}

The authors would like to thank Dr. C. Y. Wong for helpful discussions. This research was supported by the National Natural Science Foundation of China under grant 11075027.
[1] Y. Aoki, G. Endrodi, Z. Fodor, S. D. Katz, and K. K. Szabo, Nature 443, 675 (2006).

[2] N. G. Antoniou and A. S. Kapoyannis, Phys. Lett. B 563, 165 (2003).

[3] Y. Hatta and T. Ikeda, Phys. Rev. D 67, 014028 (2003).

[4] Z. Fodor and S. D. Katz, JHEP 0404, 050 (2004).

[5] R. Gavai, S. Gupta, Phys. Rev. D 71, 114014 (2005).

[6] M. Asakawa, S. A. Bass, B. Muller, and C. Nonaka, Phys. Rev. Lett. 101, 122302 (2008).

[7] S. Ejiri, Phys. Rev. D 78, 074507 (2008).

[8] G. S. F. Stephans, J. Phys. G 32, S447 (2006); G. S. F. Stephans, ibid. 35, 044050 (2008).

[9] M. M. Aggarwal et al. (STAR Collaboration), arXiv:1007.2613.

[10] M. Gazdzicki for NA49 Collaboration, invited talk at 3rd International Workshop on the Critical Point and Onset of Deconfinement, July 3-6 2006, Florence, Italy; nucl-ex/0612007

[11] M. Gazdzicki for NA61/SHINE Collaboration, J. Phys. G 36, 064039 (2009).

[12] M. Gazdzicki for NA49 and NA61/SHINE Collaborations, invited talk at Quark Matter 2011, May 23-28, 2011, Annecy, France; arXiv:1107.2345.

[13] V. Friese, Nucl. Phys. A 774, 377 (2006).

[14] K. Peters, Nucl. Phys. B (Proc. Suppl.) 154, 35 (2006); G. Rosner, ibid. 167, 77 (2007).

[15] W. F. Henning, Nucl. Phys. A 805, 502c (2008).
[16] C. Höhne, Nucl. Phys. A 830, 369c (2009); J. M. Heuser for CBM Collaboration, ibid. 563c (2009).

[17] C. Y. Wong, Introduction to High-Energy Heavy-Ion Collisions (World Scientific, Singapore, 1994), Chap. 17.

[18] U. Wiedemann, U. Heinz, Phys. Rept. 319, 145 (1999).

[19] R. M. Weiner, Phys. Rept. 327, 249 (2000).

[20] M. A. Lisa, S. Pratt, R. Soltz, and U. Wiedemann, Ann. Rev. Nucl. Part. Sci. 55, 357 (2005).

[21] S. Pratt, Phys. Rev. D 33, 1314 (1986).

[22] G. Bertsch and G. E. Brown, Phys. Rev. C 40, 1830 (1989).

[23] C. M. Hung and E. Shuryak, Phy. Rev. Lett. 75, 4003 (1995).

[24] D. H. Rischke and M. Gyulassy, Nucl. Phys. A 608, 479 (1996).

[25] S. Soff, S. A. Bass, D. H. Hardtke, and S. Y. Panitkin, Phys. Rev. Lett. 88, 072301 (2002).

[26] D. Zschiesche, H. Stöcker, W. Greiner, and S. Schramm, Phys. Rev. C 65, 064902 (2002).

[27] L. L. Yu, W. N. Zhang, and C. Y. Wong, Phys. Rev. C 78, 014908 (2008).

[28] Li-Li Yu, M. J. Efaaf, Wei-Ning Zhang, Chin. Phys. Lett. 27, 022501 (2010).

[29] G. Bertsch, M. Gong, M. Tohyama, Phys. Rev. C 37, 1896 (1988); G. Bertsch, Nucl. Phys. A 498, 173c (1989).

[30] S. Pratt, T. Csörgo, and J. Zimányi, Phys. Rev. C 42, 2646 (1990). 
[31] D. H. Rischke, nucl-th/9809044.

[32] P. F. Kolb and U. Heinz U, nucl-th/0305084.

[33] V. D. Toneev, E. G. Nikonov, B. Friman, W. Nörenberg, and K. Redlich, Eur. Phys. J. C 32, 399 (2003).

[34] D. H. Rischke, M. I. Gorenstein, H. Stöcker, and W. Greiner, Z. Phys. C 51, 485 (1991).

[35] C. M. Hung and E. Shuryak, Phys. Rev. C 57, 1891 (1998).

[36] Y. B. Ivanov, V. N. Russkikh, and V. D. Toneev, Phys. Rev. C 73, 044904 (2006).

[37] P. R. Subramanian, H. Stocker, and W. Greiner, Phys. Lett. B 173, 468 (1986).

[38] G. A. Sod, J. Fluid Mech. 83, 785 (1977).

[39] V. Schneider, J. Comput. Phys. 105, 92 (1993).

[40] D. H. Rischke, S. Bernard, and J. A. Maruhn, Nucl. Phys. A 595, 346 (1995).
[41] C. Y. Wong, J. Phys. G 29, 2151 (2003).

[42] C. Y. Wong, J. Phys. G 30, S1053 (2004).

[43] W. N. Zhang, M. J. Efaaf, C. Y. Wong, and M. Khaliliasr, Chin. Phys. Lett. 21, 1918 (2004).

[44] C. Y. Wong, AIP Conference Proc. 828, 617 (2006); hep-ph/0510258

[45] H. Bebie, P. Gerber, J. L. Goity, and H. Leutwyler, Nucl. Phys. B 378, 95 (1992).

[46] T. Hirano and K. Tsuda, Phys. Rev. C 66, 054905 (2002).

[47] J. Cleymans and K. Redlich, Phys. Rev. C 60, 054908 (1999).

[48] M. Herrmann, G. Bertsch, Phys. Rev. C 51, 328 (1995).

[49] S. Chapman, P. Scotto, and U. Heinz, Phys. Rev. Lett. 74, 4400 (1995). 